\title{
A regularization of Zubov's equation for robust domains of attraction ${ }^{\star}$
}

\author{
Fabio Camilli ${ }^{1}$, Lars Grüne ${ }^{2}$, and Fabian Wirth ${ }^{3 \star \star}$ \\ 1 Dip. di Energetica, Fac. di Ingegneria, Università de l'Aquila, \\ 67040 Roio Poggio (AQ), Italy, camilli@axcasp.caspur.it \\ 2 Fachbereich Mathematik, J.W. Goethe-Universität, Postfach 1119 32, \\ 60054 Frankfurt a.M., Germany, gruene@math.uni-frankfurt.de \\ 3 Center for Technomathematics, University of Bremen \\ 28334 Bremen, Germany, fabian@math.uni-bremen.de
}

\begin{abstract}
We derive a method for the computation of robust domains of attraction based on a recent generalization of Zubov's theorem on representing robust domains of attraction for perturbed systems via the viscosity solution of a suitable partial differential equation. While a direct discretization of the equation leads to numerical difficulties due to a singularity at the stable equilibrium, a suitable regularization enables us to apply a standard discretization technique for HamiltonJacobi-Bellman equations. We present the resulting fully discrete scheme and show a numerical example.
\end{abstract}

\section{Introduction}

The domain of attraction of an asymptotically stable fixed point has been one of the central objects in the study of continuous dynamical systems. The knowledge of this object is important in many applications modeled by those systems like e.g. the analysis of power systems [1] and turbulence phenomena in fluid dynamics $[2,8,17]$. Several papers and books discuss theoretical $[19,20,5,12]$ as well as computational aspects $[18,13,1,9]$ of this problem.

Taking into account that usually mathematical models of complex systems contain model errors and that exogenous perturbations are ubiquitous it is natural to consider systems with deterministic time varying perturbations and look for domains of attraction that are robust under all these perturbations. Here we consider systems of the form

$$
\dot{x}(t)=f(x(t), a(t)), \quad x \in \mathbb{R}^{n}
$$

\footnotetext{
* Research supported by the TMR Networks "Nonlinear Control Network" and "Viscosity Solutions and their applications", and the DFG Priority Research Program "Ergodentheorie, Analysis und effiziente Simulation dynamischer Systeme"

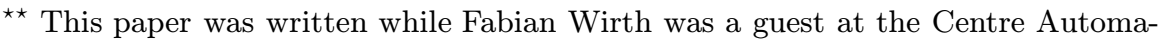
tique et Systèmes, Ecole des Mines de Paris, Fontainebleau, France. The hospitality of all the members of the centre is gratefully acknowledged.
} 
where $a(\cdot)$ is an arbitrary measurable function with values in some compact set $A \subset \mathbb{R}^{m}$. Under the assumption that $x^{*} \in \mathbb{R}^{n}$ is a locally exponentially stable fixed point for all admissible perturbation functions $a(\cdot)$ we try to find the set of points which are attracted to $x^{*}$ for all admissible $a(\cdot)$.

This set has been considered e.g. in [14,15,4,7]. In particular, in [14] and [7] numerical procedures based on optimal control techniques for the computation of robust domains of attraction are presented. The techniques in these papers have in common that a numerical approximation of the optimal value function of a suitable optimal control problem is computed such that the robust domain of attraction is characterized by a suitable sublevel set of this function. Whereas the method in [14] requires the numerical solution of several Hamilton-Jacobi-Bellman equations (and is thus very expensive) the method in [7] needs just one such solution, but requires some knowledge about the local behavior around $x^{*}$ in order to avoid discontinuities in the optimal value functions causing numerical problems.

In this paper we use a similar optimal control technique, but start from recent results in [4] where the classical equation of Zubov [20] is generalized to perturbed systems. Under very mild conditions on the problem data this equation admits a continuous or even Lipschitz viscosity solution. The main problem in a numerical approximation is the inherent singularity of the equation at the fixed point which prevents the direct application of standard numerical schemes. Here we propose a regularization of this equation such that the classical schemes [6] and adaptive gridding techniques [11] are applicable without losing the main feature of the solution, i.e. the sublevel set characterization of the robust domain of attraction. It might be worth noting that in particular our approach is applicable to the classical Zubov equation (i.e. for unperturbed systems) and hence provides a way to compute domains of attraction also for unperturbed systems.

This paper is organized as follows: In Section 2 we give the setup and collect some facts about robust domains of attraction. In Section 3 we summarize the needed results from [4] on the generalization of Zubov's equation for perturbed system. In Section 4 we introduce the regularization technique and formulate the numerical scheme, and finally, in Section 5 we show a numerical example.

\section{Robust domains of attraction}

We consider systems of the following form

$$
\left\{\begin{array}{l}
\dot{x}(t)=f(x(t), a(t)), \quad t \in[0, \infty), \\
x(0)=x_{0},
\end{array}\right.
$$

with solutions denoted by $x\left(t, x_{0}, a\right)$. Here $a(\cdot) \in \mathcal{A}=L^{\infty}([0,+\infty), A)$ and $A$ is a compact subset of $\mathbb{R}^{m}, f$ is continuous and bounded in $\mathbb{R}^{n} \times A$ and 
Lipschitz in $x$ uniformly in $a \in A$. Furthermore, we assume that the fixed point $x=0$ is singular, that is $f(0, a)=0$ for any $a \in A$.

We assume that the singular point 0 is uniformly locally exponentially stable for the system (1), i.e.

$$
\begin{aligned}
& \text { there exist constants } C, \sigma, r>0 \text { such that } \\
& \left\|x\left(t, x_{0}, a\right)\right\| \leq C e^{-\sigma t}\left\|x_{0}\right\| \text { for any } x_{0} \in B(0, r) \text { and any } a \in A .
\end{aligned}
$$

The following sets describe domains of attraction for the equilibrium $x=0$ of the system (1).

Definition 1. For the system (1) satisfying (H1) we define the robust domain of attraction as

$$
\mathcal{D}=\left\{x_{0} \in \mathbb{R}^{n}: x\left(t, x_{0}, a\right) \rightarrow 0 \text { as } t \rightarrow+\infty \text { for any } a \in \mathcal{A}\right\},
$$

and the uniform robust domain of attraction by

$$
\mathcal{D}_{0}=\left\{x_{0} \in \mathbb{R}^{n}: \begin{array}{l}
\text { there exists a function } \beta(t) \rightarrow 0 \text { as } t \rightarrow \infty \\
\text { s.th. }\left\|x\left(t, x_{0}, a\right)\right\| \leq \beta(t) \text { for all } t>0, a \in \mathcal{A}
\end{array}\right\} .
$$

For a collection of properties of (uniform) robust domains of attraction we refer to [4, Proposition 2.4]. There it is shown in particular, that $\mathcal{D}_{0}$ is an open, connected and invariant set, and that the inclusion $\mathcal{D} \subset \operatorname{cl} \mathcal{D}_{0}$ holds.

\section{Zubov's method for robust domains of attraction}

In this section we discuss the following partial differential equation

$$
\inf _{a \in A}\{-D v(x) f(x, a)-(1-v(x)) g(x, a)\}=0 \quad x \in \mathbb{R}^{n}
$$

whose solution will turn out to characterize the uniform robust domain of attraction $\mathcal{D}_{0}$. This equation is a straightforward generalization of Zubov's equation [20]. In this generality, however, in order to obtain a meaningful result about solutions we have to work within the framework of viscosity solutions, which we recall for the convenience of the reader (for details about this theory we refer to [3]).

Definition 2. Given an open subset $\Omega$ of $\mathbb{R}^{n}$ and a continuous function $H: \Omega \times \mathbb{R} \times \mathbb{R}^{n} \rightarrow \mathbb{R}$, we say that a lower semicontinuous (l.s.c.) function $u: \Omega \rightarrow \mathbb{R}$ (resp. an upper semicontinuous (u.s.c.) function $v: \Omega \rightarrow \mathbb{R}$ ) is a viscosity supersolution (resp. subsolution) of the equation

$$
H(x, u, D u)=0 \quad x \in \Omega
$$

if for all $\phi \in C^{1}(\Omega)$ and $x \in \operatorname{argmin}_{\Omega}(u-\phi)$ (resp., $\left.x \in \operatorname{argmax}_{\Omega}(v-\phi)\right)$ we have

$$
H(x, u(x), D \phi(x)) \geq 0 \quad(\text { resp., } H(x, v(x), D \phi(x)) \leq 0) .
$$

A continuous function $u: \Omega \rightarrow \mathbb{R}$ is said to be a viscosity solution of (3) if $u$ is a viscosity supersolution and a viscosity subsolution of (3). 
We now introduce the value function of a suitable optimal control problem related to $(2)$.

Consider the functional $G: \mathbb{R}^{n} \times \mathcal{A} \rightarrow \mathbb{R} \cup\{+\infty\}$ and the optimal value function $v$ given by

$$
G^{\infty}(x, a):=\int_{0}^{+\infty} g(x(t), a(t)) d t \quad \text { and } \quad v(x):=\sup _{a \in \mathcal{A}} 1-e^{-G^{\infty}(x, a)}
$$

where the function $g: \mathbb{R}^{n} \times A \rightarrow \mathbb{R}$ is supposed to be continuous and satisfies

(i) For any $a \in A, g(0, a)=0$ and $g(x, a)>0$ for $x \neq 0$.

(ii) There exists a constant $g_{0}>0$ such that $\inf _{x \notin B(0, r), a \in A} g(x, a) \geq g_{0}$.

(iii) For every $R>0$ there exists a constant $L_{R}$ such that $\|g(x, a)-g(y, a)\| \leq L_{R}\|x-y\|$ for all $\|x\|,\|y\| \leq R$ and all $a \in A$.

Since $g$ is nonnegative it is immediate that $v(x) \in[0,1]$ for all $x \in \mathbb{R}^{n}$. Furthermore, standard techniques from optimal control (see e.g. [3, Chapter III]) imply that $v$ satisfies a dynamic programming principle, i.e. for each $t>0$ we have

$$
v(x)=\sup _{a \in \mathcal{A}}\{(1-G(x, t, a))+G(x, t, a) v(x(t, x, a))\}
$$

with

$$
G(t, x, a):=\exp \left(-\int_{0}^{t} g(x(\tau, x, a), a(\tau)) d \tau\right) .
$$

Furthermore, a simple application of the chain rule shows

$$
(1-G(x, t, a))=\int_{0}^{t} G(x, \tau, a) g(x(\tau, x, a), a(\tau)) d \tau
$$

implying (abbreviating $G(t)=G(x, t, a)$ )

$$
v(x)=\sup _{a \in \mathcal{A}}\left\{\int_{0}^{t} G(\tau) g(x(\tau, x, a), a(\tau)) d \tau+G(t) v(x(t, x, a))\right\}
$$

The next proposition shows the relation between $\mathcal{D}_{0}$ and $v$, and the continuity of $v$. For the proof see [4, Proposition 3.1]

Proposition 1. Assume (H1), (H2). Then

(i) $v(x)<1$ if and only if $x \in \mathcal{D}_{0}$.

(ii) $v(0)=0$ if and only if $x=0$.

(iii) $v$ is continuous on $\mathbb{R}^{n}$.

(iv) $v(x) \rightarrow 1$ for $x \rightarrow x_{0} \in \partial \mathcal{D}_{0}$ and for $\|x\| \rightarrow \infty$. 
We now turn to the relation between $v$ and equation (2). Recalling that $v$ is locally bounded on $\mathbb{R}^{n}$ an easy application of the dynamic programming principle (5) (cp. [3, Chapter III]) shows that and $v$ is a viscosity solution of (2). The more difficult part is to obtain uniqueness of the solution, since equation (2) exhibits a singularity at the origin.

In order to prove the following uniqueness result we use super- and suboptimality principles, which essentially follow from Soravia [16, Theorem 3.2 (i)], see $[4$, Section 3$]$ for details.

Theorem 1. Consider the system (1) and a function $g: \mathbb{R}^{n} \times A \rightarrow \mathbb{R}$ such that (H1) and (H2) are satisfied. Then (2) has a unique bounded and continuous viscosity solution $v$ on $\mathbb{R}^{n}$ satisfying $v(0)=0$.

This function coincides with $v$ from (4). In particular the characterization $\mathcal{D}_{0}=\left\{x \in \mathbb{R}^{n} \mid v(x)<1\right\}$ holds.

We also obtain the following local version of this result.

Theorem 2. Consider the system (1) and a function $g: \mathbb{R}^{n} \times A \rightarrow \mathbb{R}$. Assume (H1) and (H2). Let $\mathcal{O} \subset \mathbb{R}^{n}$ be an open set containing the origin, and let $v: \mathrm{clO} \rightarrow \mathbb{R}$ be a bounded and continuous function which is a viscosity solution of (2) on $\mathcal{O}$ and satisfies $v(0)=0$ and $v(x)=1$ for all $x \in \partial \mathcal{O}$.

Then $v$ coincides with the restriction $\left.v\right|_{\mathcal{O}}$ of the function $v$ from (4). In particular the characterization $\mathcal{D}_{0}=\left\{x \in \mathbb{R}^{n} \mid v(x)<1\right\}$ holds.

We end this section by stating several additional properties of $v$ as proved in $[4$, Sections 4 and 5].

Theorem 3. Assume (H1) and (H2) and consider the unique viscosity solution $v$ of (2) with $v(0)=0$. Then the following statements hold.

(i) The function $v$ is a robust Lyapunov function for the system (1), i.e.

$$
v\left(x\left(t, x_{0}, a(\cdot)\right)\right)-v\left(x_{0}\right)<0
$$

for all $x_{0} \in \mathcal{D}_{0} \backslash\{0\}$, all $t>0$ and all $a(\cdot) \in \mathcal{A}$.

(ii) If $f(\cdot, a)$ and $g(\cdot, a)$ are uniformly Lipschitz continuous in $\mathbb{R}^{n}$, with constants $L_{f}, L_{g}>0$ uniformly in $a \in A$, and if there exists a neighborhood $N$ of the origin such that for all $x, y \in N$ the inequality

$$
|g(x, a)-g(y, a)| \leq K \max \{\|x\|,\|y\|\}^{s}\|x-y\|
$$

holds for some $K>0$ and $s>L_{f} / \sigma$ with $\sigma>0$ given by (H1), then the function $v$ is Lipschitz continuous in $\mathbb{R}^{n}$ for all $g$ with $g_{0}>0$ from (H2) sufficiently large.

(iii) If $f(x, A)$ is convex for all $x \in \mathbb{R}^{n}$ and $B \subset \mathcal{D}_{0}$ satisfies $\operatorname{dist}\left(B, \partial \mathcal{D}_{0}\right)>0$, then there exists a function $g: \mathbb{R}^{n} \rightarrow \mathbb{R}$ satisfying (H2) such that the solution $v$ of (2) is $C^{\infty}$ on a neighborhood of $B$. 


\section{Numerical solution}

A first approach to solve equation (2) is to directly adopt the first order numerical scheme from [6] to this equation. Considering a bounded domain $\Omega$ and a simplicid grid $\Gamma$ with edges $x_{i}$ covering $\operatorname{cl} \Omega$ this results in solving

$$
\tilde{v}\left(x_{i}\right)=\max _{a \in A}\left\{\left(1-h g\left(x_{i}, a\right)\right) \tilde{v}\left(x_{i}+h f\left(x_{i}, a\right)\right)+h g\left(x_{i}, a\right)\right\}
$$

where $\tilde{v}$ is continuous and affinely linear on each simplex in the grid and satisfies $\tilde{v}(0)=0$ (assuming, of course, that 0 is a node of the grid) and $\tilde{v}\left(x_{i}\right)=1$ for all $x_{i} \in \partial \Omega$. Unfortunately, since also (8) has a singularity in 0 the fixed point argument used in [6] fails here and hence convergence is not guaranteed. In fact, it is easy to see that in the situation of Figure 1 (showing one trajectory and the simplices surrounding the fixed point 0 in a two-dimensional example) the piecewise linear function $\tilde{v}$ with

$$
\tilde{v}\left(x_{i}\right)=\left\{\begin{array}{l}
1, x_{i} \neq 0 \\
0, x_{i}=0
\end{array}\right.
$$

satisfies (8), since for all nodes $x_{i} \neq 0$ the value $x_{i}+h f\left(x_{i}, a\right)$ lies in a simplex with nodes $x_{j} \neq 0$, hence $\tilde{v}\left(x_{i}+h f\left(x_{i}, a\right)\right)=1$ implying

$$
\left(1-h g\left(x_{i}, a\right)\right) \tilde{v}\left(x_{i}+h f\left(x_{i}, a\right)\right)+h g\left(x_{i}, a\right)=1=\tilde{v}\left(x_{i}\right),
$$

i.e. (8). As this situation may occur for arbitrarily fine grids indeed convergence is not guaranteed.

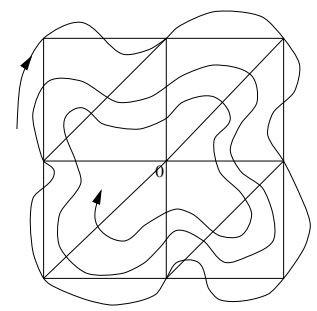

Fig. 1. A situation of non-convergence

In order to ensure convergence we will therefore have to use a regularization of (2). The main idea in this is to change (2) in such a way that the "discount rate" (i.e. the factor $g(x)$ in front of the zero order term $v(x)$ ) becomes strictly positive, and thus the singularity disappears. To this end consider some parameter $\varepsilon>0$ and consider the function

$$
g_{\varepsilon}(x, a)=\max \{g(x, a), \varepsilon\} .
$$

Using this $g_{\varepsilon}$ we approximate (2) by

$$
\inf _{a \in A}\left\{-D v(x) f(x, a)-g(x, a)+v(x) g_{\varepsilon}(x, a)\right\}=0 \quad x \in \mathbb{R}^{n} .
$$


The following proposition summarizes some properties of (9). We state it in a global version on $\mathbb{R}^{n}$, the analogous statements hold in the situation of Theorem 2.

Proposition 2. Let the assumptions of Theorem 1 hold and let $v$ be the unique solution of (2) with $v(0)=0$. Then for each $\varepsilon>0$ equation (9) has a unique continuous viscosity solution $v_{\varepsilon}$ with the following properties.

(i) $v_{\varepsilon}(x) \leq v(x)$ for all $x \in \mathbb{R}^{n}$

(ii) $v_{\varepsilon} \rightarrow v$ uniformly in $\mathbb{R}^{n}$ as $\varepsilon \rightarrow 0$

(iii) If $\varepsilon<g_{0}$ from (H2)(ii) then the characterization $\mathcal{D}_{0}=\left\{x \in \mathbb{R}^{n} \mid v_{\varepsilon}(x)<\right.$ $1\}$ holds

(iv) If $f(\cdot, a)$ and $g(\cdot, a)$ are uniformly Lipschitz on $\mathcal{D}_{0}$ (uniformly in A with Lipschitz constants $L_{f}$ and $L_{g}$ ) and $g$ is bounded on $\mathcal{D}_{0}$ and satisfies the inequalities

$$
|g(x, a)-g(y, a)| \leq K \max \{\|x\|,\|y\|\}^{s}\|x-y\|
$$

for all $x, y \in B(0, C r)$ and

$$
|g(x, a)| \geq g_{1}>L_{f}
$$

for all $x \notin B(0, r / 2)$ with $C, \sigma$ and $r$ from (H1), then the function $v_{\varepsilon}$ is uniformly Lipschitz on $\mathbb{R}^{n}$.

Proof: Since the discount rate in (9) is strictly positive it follows by standard viscosity solution arguments [3, Chapter III] that there exists a unique solution $v_{\varepsilon}$ which furthermore for all $t \geq 0$ satisfies the following dynamic programming principle

$$
v_{\varepsilon}(x)=\sup _{a \in \mathcal{A}}\left\{\int_{0}^{t} G_{\varepsilon}(\tau) g(x(\tau, x, a), a(\tau)) d \tau+G_{\varepsilon}(t) v_{\varepsilon}(x(t, x, a))\right\}
$$

with

$$
G_{\varepsilon}(t)=G_{\varepsilon}(x, t, a):=\exp \left(-\int_{0}^{t} g_{\varepsilon}(x(\tau, x, a), a(\tau)) d \tau\right) .
$$

Since $v$ satisfies the same principle ( 7$)$ with $G(x, t, a) \geq G_{\varepsilon}(x, t, a)$ by (6) and $g>0$ the stated inequality (i) follows.

In order to see (ii) observe that the continuity of $g$ and $v$ implies that for each $\delta>0$ there exists $\varepsilon>0$ such that

$$
\left\{x \in \mathbb{R}^{n} \mid g_{\varepsilon}(x, a) \geq g(x, a) \text { for some } a \in A\right\} \subset\left\{x \in \mathbb{R}^{n} \mid v(x) \leq \delta\right\} .
$$

Now fix $\delta>0$ and consider the corresponding $\varepsilon>0$. Let $x \in \mathbb{R}^{n}$ and pick some $\gamma>0$ and a control $a_{\gamma} \in \mathcal{A}$ such that

$$
v(x) \leq \int_{0}^{\infty} G\left(x, \tau, a_{\gamma}\right) g\left(x\left(\tau, x, a_{\gamma}\right), a_{\gamma}(\tau)\right) d \tau+\gamma .
$$


Now let $T \geq 0$ be the (unique) time with $v\left(x\left(T, x, a_{\gamma}\right)\right)=\delta$. Abbreviating $G(\tau)=G\left(x, \tau, a_{\gamma}\right)$ and $G_{\varepsilon}(\tau)=G_{\varepsilon}\left(x, \tau, a_{\gamma}\right)$ we can conclude that

$$
\begin{aligned}
& v(x)-v_{\varepsilon}(x)-\gamma \\
& \leq \int_{0}^{\infty}\left(G(\tau) g\left(x\left(\tau, x, a_{\gamma}\right), a_{\gamma}(\tau)\right)-G_{\varepsilon}(\tau) g\left(x\left(\tau, x, a_{\gamma}\right), a_{\gamma}(\tau)\right)\right) d \tau \\
& \leq \int_{0}^{T} \underbrace{\left(G(\tau) g\left(x\left(\tau, x, a_{\gamma}\right), a_{\gamma}(\tau)\right)-G_{\varepsilon}(\tau) g\left(x\left(\tau, x, a_{\gamma}\right), a_{\gamma}(\tau)\right)\right)}_{=0} d \tau \\
& \quad+G(T) v\left(x\left(T, x, a_{\gamma}\right)\right) \leq \delta .
\end{aligned}
$$

Since $\gamma>0$ and $x \in \mathbb{R}^{n}$ were arbitrary this shows (ii).

To prove (iii) let $\varepsilon<g_{0}$. Then for all $x \notin \mathcal{D}_{0}$ and all $T>0$ there exists $a \in \mathcal{A}$ such that $G(x, t, a)=G_{\varepsilon}(x, t, a)$ for all $t \in[0, T]$ which immediately implies $\mathcal{D}_{0}=\left\{x \in \mathbb{R}^{n} \mid v_{\varepsilon}(x)<1\right\}$.

In order to see (iv) first note that (10) holds for $g_{\varepsilon}$ for all $\varepsilon \geq 0$ (with the convention $g_{0}=g$ ). Hence by straightforward integration using the exponential stability and (10) we can estimate

$$
\left|\int_{0}^{t} g_{\varepsilon}(x(\tau, x, a), a(\tau)) d \tau-\int_{0}^{t} g_{\varepsilon}(x(\tau, y, a), a(\tau)) d \tau\right| \leq L_{0}\|x-y\|
$$

for all $x, y \in B(0, r)$ and some $L_{0}>0$ independent of $\varepsilon$ and $a$, which also implies

$$
\left|G_{\varepsilon}(x, t, a)-G_{\varepsilon}(y, t, a)\right| \leq L_{0}\|x-y\|
$$

for all $t \geq 0$ and consequently

$$
\begin{aligned}
& \sup _{a \in \mathcal{A}} \mid \int_{0}^{\infty} G_{\varepsilon}(x, \tau, a) g(x(\tau, x, a), a(\tau)) d \tau \\
& \quad-\int_{0}^{\infty} G_{\varepsilon}(y, \tau, a) g(x(\tau, y, a), a(\tau)) d \tau \mid \\
& \leq \sup _{a \in \mathcal{A}} \int_{0}^{\infty}\left|G_{\varepsilon}(x, \tau, a)-G_{\varepsilon}(y, \tau, a)\right| \underbrace{g(x(\tau, x, a), a(\tau))}_{\leq L_{g} C e^{-\sigma \tau}\|x\|} d \tau \\
& \quad+\int_{0}^{\infty} \underbrace{G_{\varepsilon}(y, \tau, a)}_{\leq 1}|g(x(\tau, x, a), a(\tau))-g(x(\tau, y, a), a(\tau))| d \tau \\
& \leq L_{1}\|x-y\|
\end{aligned}
$$

for some suitable $L_{1}>0$ and all $x, y \in B(0, r)$, implying in particular

$$
\left|v_{\varepsilon}(x)-v_{\varepsilon}(y)\right| \leq L_{1}\|x-y\| .
$$

For all $t>0$ with $x(s, x, a) \notin B(0, r / 2)$ and $x(s, y, a) \notin B(0, r / 2)$ for all $s \in[0, t]$ we can estimate

$$
\left|G_{\varepsilon}(x, t, a)\right| \leq e^{-t g_{1}}, \quad\left|G_{\varepsilon}(y, t, a)\right| \leq e^{-t g_{1}}
$$


and using $\left|e^{-a}-e^{-b}\right| \leq \max \left\{e^{-a}, e^{-b}\right\}|a-b|$ it follows

$$
\begin{aligned}
& \left|G_{\varepsilon}(x, t, a)-G_{\varepsilon}(y, t, a)\right| \\
& \leq e^{-t g_{1}} \int_{0}^{t}\left|g_{\varepsilon}(x(\tau, x, a), a(\tau))-g_{\varepsilon}(x(\tau, y, a), a(\tau))\right| d \tau \\
& \leq e^{-t g_{1}} \int_{0}^{t} L_{g} e^{\tau L_{f}}\|x-y\| d \tau \\
& \leq e^{-t g_{1}} \frac{L_{g}}{L_{f}} e^{t L_{f}}\|x-y\|=e^{t\left(L_{f}-g_{1}\right)} \frac{L_{g}}{L_{f}}\|x-y\| .
\end{aligned}
$$

Now define $T(x, a):=\inf \{t>0: x(t, x, a) \in B(0, r / 2)\}$. Then by continuous dependence on the initial value (recall that $f$ is Lipschitz in $x$ uniformly in $a \in A)$ for each $x \in \mathcal{D}_{0} \backslash B(0, r)$ there exists a neighborhood $\mathcal{N}(x)$ such that $x(t(x, a), y, a) \in B(0, r)$ and $x(t(y, a), x, a) \in B(0, r)$ for all $y \in \mathcal{N}(x)$ and all $a \in \mathcal{A}$. Pick some $x \in \mathcal{D}_{0} \backslash B(0, r)$ and some $y \in \mathcal{N}(x)$. Then for each $\gamma>0$ we find $a_{\gamma} \in \mathcal{A}$ such that

$$
\begin{aligned}
& \left|v_{\varepsilon}(x)-v_{\varepsilon}(y)\right|-\gamma \\
& \leq \mid \int_{0}^{\infty} G_{\varepsilon}\left(x, \tau, a_{\gamma}\right) g\left(x\left(\tau, x, a_{\gamma}\right), a_{\gamma}(\tau)\right) d \tau \\
& \quad-\int_{0}^{\infty} G_{\varepsilon}\left(y, \tau, a_{\gamma}\right) g\left(x\left(\tau, y, a_{\gamma}\right), a_{\gamma}(\tau)\right) d \tau \mid .
\end{aligned}
$$

Now fix some $\gamma>0$ and let $T:=\min \left\{T\left(x, a_{\gamma}\right), T\left(y, a_{\gamma}\right)\right\}$. Abbreviating $x(t)=x\left(t, x, a_{\gamma}\right)$ and $y(t)=x\left(t, y, a_{\gamma}\right)$ we can conclude that $x(T) \in B(0, r)$ and $y(T) \in B(0, r)$. Hence we can continue

$$
\begin{aligned}
& \left|v_{\varepsilon}(x)-v_{\varepsilon}(y)\right|-\gamma \\
& \leq\left|\int_{0}^{T} G_{\varepsilon}\left(x, \tau, a_{\gamma}\right) g\left(x(\tau), a_{\gamma}(\tau)\right) d \tau-\int_{0}^{T} G_{\varepsilon}\left(y, \tau, a_{\gamma}\right) g\left(y(\tau), a_{\gamma}(\tau)\right) d \tau\right| \\
& +G_{\varepsilon}\left(x, T, a_{\gamma}\right) \mid \int_{0}^{\infty} G_{\varepsilon}\left(x(T), \tau, a_{\gamma}(T+\cdot)\right) g\left(x(T+\tau), a_{\gamma}(T+\tau)\right) d \tau \\
& -\int_{0}^{\infty} G_{\varepsilon}\left(y(T), \tau, a_{\gamma}(T+\cdot)\right) g\left(y(T+\tau), a_{\gamma}(T+\tau)\right) d \tau \mid \\
& \leq \int_{0}^{T}\left|G_{\varepsilon}\left(x, \tau, a_{\gamma}\right)-G_{\varepsilon}\left(y, \tau, a_{\gamma}\right)\right| g\left(x(\tau), a_{\gamma}(\tau)\right) d \tau \\
& +\int_{0}^{T} G_{\varepsilon}\left(y, \tau, a_{\gamma}\right)\left|g\left(x\left(\tau, x, a_{\gamma}\right), a_{\gamma}(\tau)\right)-g\left(y(\tau), a_{\gamma}(\tau)\right)\right| d \tau \\
& +e^{-g_{1} T} e^{L_{f} T} L_{1}\|x-y\| \\
& \leq \int_{0}^{T}\left|G_{\varepsilon}\left(x, \tau, a_{\gamma}\right)-G_{\varepsilon}\left(y, \tau, a_{\gamma}\right)\right| \underbrace{g\left(x(\tau), a_{\gamma}(\tau)\right)}_{\leq \sup _{x \in \mathcal{D}_{0}, a \in A} g(x, a)=: g^{*}} d \tau
\end{aligned}
$$




$$
\begin{aligned}
& \quad+\int_{0}^{T} e^{-\tau g_{1}} \underbrace{\left|g\left(x(\tau), a_{\gamma}(\tau)\right)-g\left(y(\tau), a_{\gamma}(\tau)\right)\right|}_{\leq L_{g} e^{t L_{f}}\|x-y\|} d \tau \\
& \quad+L_{1}\|x-y\| \\
& \leq\left(g^{*} \frac{L_{g}}{L_{f}\left(g_{1}-L_{f}\right)}+\frac{L_{g}}{g_{1}-L_{f}}+L_{1}\right)\|x-y\|
\end{aligned}
$$

since $g_{1}>L_{f}$. Here the first inequality follows by splitting up the integrals using the triangle inequality, the second follows by the triangle inequality for the first term and using $x(T), y(T) \in B(0, r),\|x(T)-y(T)\| \leq e^{L_{f} T}$, and (15) for the second term, and the third and fourth inequality follow from (16) and (17).

Since $\gamma>0$ was arbitrary the Lipschitz property follows on $\mathcal{D}_{0}$, thus also on cl $\mathcal{D}_{0}$ and consequently on the whole $\mathbb{R}^{n}$ since $v_{\varepsilon} \equiv 1$ on $\mathbb{R}^{n} \backslash \mathcal{D}_{0}$.

Remark 1. Note that in general the solution $v_{\varepsilon}$ is not a robust Lyapunov function for the origin of (1) anymore. More precisely, we can only ensure decrease of $v_{\varepsilon}$ along trajectories $x\left(t, x_{0}, a\right)$ as long as $g\left(x\left(t, x_{0}, a\right), a(t)\right)>\varepsilon$, i.e. outside the region where the regularization is effective. Hence although many properties of $v$ are preserved in this regularization, some are nevertheless lost.

We now apply the numerical scheme from [6] to (9). Thus we end up with

$$
\tilde{v}_{\varepsilon}\left(x_{i}\right)=\max _{a \in A}\left\{\left(1-h g_{\varepsilon}\left(x_{i}, a\right)\right) \tilde{v}_{\varepsilon}\left(x+h f\left(x_{i}, a\right)\right)+h g\left(x_{i}, a\right)\right\}
$$

where again $\tilde{v}_{\varepsilon}$ is continuous and affinely linear on each simplex in the grid and satisfies $\tilde{v}_{\varepsilon}(0)=0$ and $\tilde{v}_{\varepsilon}\left(x_{i}\right)=1$ for all $x_{i} \in \partial \Omega$.

A straightforward modification of the arguments in [3,6] yields that there exists a unique solution $\tilde{v}_{\varepsilon}$ converging to $v_{\varepsilon}$ as $h$ and the size of the simplices tends to 0 . Note that the adaptive gridding techniques from [11] also apply to this scheme, and that a number of different iterative solvers for (18) are available, see e.g. $[6,10,11]$.

Remark 2. The numerical examples show good results also in the case where we cannot expect a globally Lipschitz continuous solution $v_{\varepsilon}$ of (9). The main reason for this seems to be that in any case $v_{\varepsilon}$ is locally Lipschitz on $\mathcal{D}_{0}$. In order to explain this observation in a rigorous way a thorough analysis of the numerical error is currently under investigation.

\section{A numerical example}

We illustrate our algorithm with a model adapted from [17]. Consider

$$
\dot{x}=\left(\begin{array}{cc}
-1 / 25 & 1 \\
0 & -2 / 25
\end{array}\right) x+\|x\|\left(\begin{array}{cc}
0 & -1 \\
1 & 0
\end{array}\right) x+\left(\begin{array}{c}
0 \\
a x_{1} x_{2}
\end{array}\right)
$$


where $x=\left(x_{1}, x_{2}\right)^{T} \in \mathbb{R}^{2}$. The unperturbed equation (i.e. with $a=0$ ) is introduced in order to explain the existence of turbulence in a fluid flow with Reynolds number $R=25$ despite the stability of the linearization at the laminar solution. In [17] simulations are made in order to estimate the domain of attraction of the locally stable equilibrium at the origin. Here we compute it entirely in a neighborhood of 0 , and in addition determine the effect of the perturbation term $a x_{1} x_{2}$ for time varying perturbation with different ranges $A$. Figure 2 shows the corresponding results obtained with the fully discrete scheme (18), setting $g(x, a)=\|x\|^{2}, \varepsilon=10^{-10}, h=1 / 20$. The grid was constructed adaptively using the techniques from [11] with a final number of about 20000 nodes. Note that due to numerical errors in the approximate solution it is not reasonable to take the "exact" sublevel sets $\tilde{v}_{\varepsilon}(x)<1$, instead some "security factor" has to be added. The domains shown in the figures are the sublevel sets $\tilde{v}_{\varepsilon}(x) \leq 0.95$.

a)

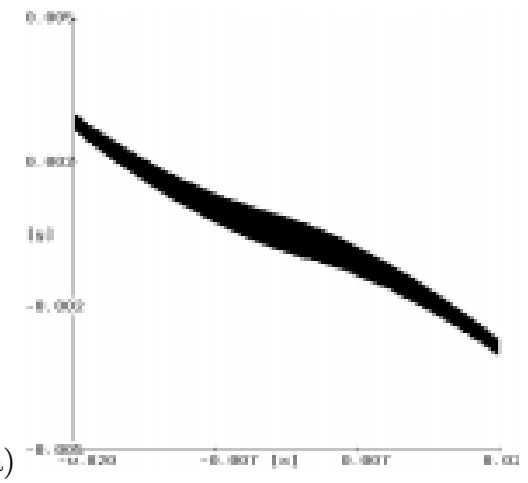

c)

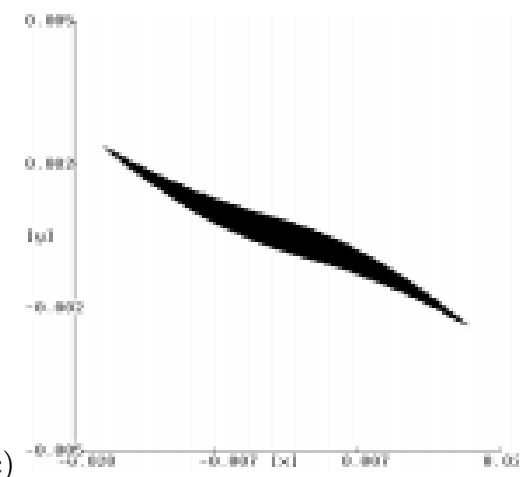

b)

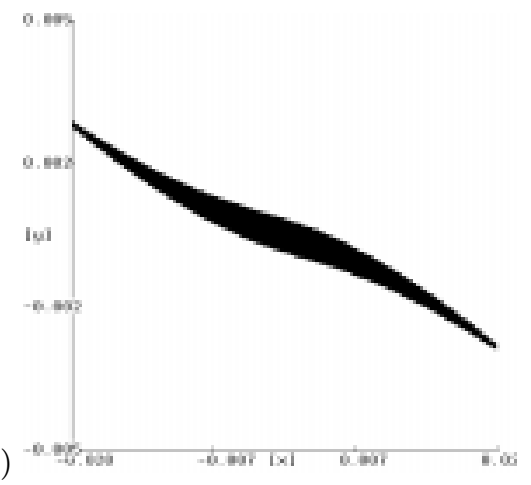

d)

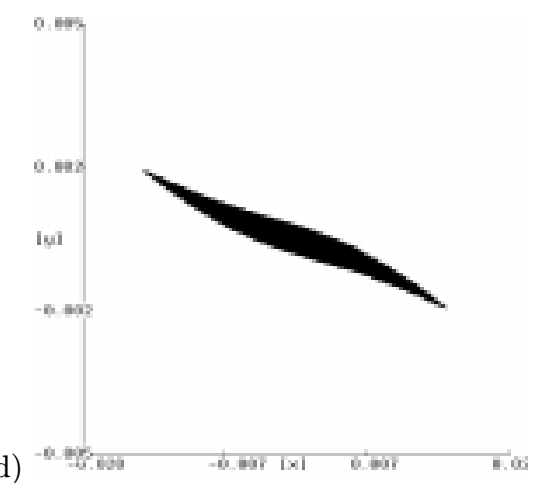

Fig. 2. Approximation of $\mathcal{D}_{0}$ for a) $A=\{0\}$, b) $A=[-1,1]$, c) $A=[-2,2]$, and d) $A=[-3,3]$ 


\section{References}

1. M. Abu Hassan and C. Storey, Numerical determination of domains of attraction for electrical power systems using the method of Zubov. Int. J. Control 34 (1981), 371-381.

2. J.S. Baggett and L.N. Trefethen, Low-dimensional Models of Subcritical Transition to Turbulence. Physics of Fluids 9 (1997), 1043-1053.

3. M. Bardi and I. Capuzzo Dolcetta, Optimal Control and Viscosity Solutions of Hamilton-Jacobi-Bellman equations, Birkhäuser, Boston, 1997.

4. F. Camilli, L. Grüne, and F. Wirth. A Generalization of Zubov's method to perturbed systems. Preprint 24/99, DFG-Schwerpunkt "Ergodentheorie, Analysis und effiziente Simulation dynamischer Systeme", submitted.

5. H.-D. Chiang, M. Hirsch, and F. Wu. Stability regions of nonlinear autonomous dynamical systems. IEEE Trans. Auto. Control 33 (1988), 16-27.

6. M. Falcone. Numerical solution of dynamic programming equations. Appendix A in: M. Bardi and I. Capuzzo Dolcetta, Optimal Control and Viscosity Solutions of Hamilton-Jacobi-Bellman equations, Birkhäuser, Boston, 1997.

7. M. Falcone, L. Grüne and F. Wirth. A maximum time approach to the computation of robust domains of attraction. Proc. EQUADIFF 99, Berlin, to appear.

8. T. Gebhardt and S. Großmann. Chaos transition despite linear stability. Phys. Rev. E 50 (1994), 3705-3711.

9. R. Genesio, M. Tartaglia, and A. Vicino. On the estimation of asymptotic stability regions: State of the art and new proposals. IEEE Trans. Auto. Control 30 (1985), 747-755.

10. R.L.V. Gonzáles and C.A. Sagastizábal, Un algorithme pour la résolution rapide d'équations discrètes de Hamilton-Jacobi-Bellman. C. R. Acad. Sci., Paris, Sér. I 311 (1990), 45-50.

11. L. Grüne. An adaptive grid scheme for the discrete Hamilton-Jacobi-Bellman equation. Numer. Math. 75 (1997), 319-337.

12. W. Hahn, Stability of Motion, Springer-Verlag, Berlin, 1967.

13. N.E. Kirin, R.A. Nelepin and V.N. Bajdaev, Construction of the attraction region by Zubov's method. Differ. Equations 17 (1982), 871-880.

14. A.D.B. Paice and F. Wirth. Robustness analysis of domains of attraction of nonlinear systems, Proceedings of the Mathematical Theory of Networks and Systems MTNS98, pages 353 - 356, Padova, Italy, 1998.

15. A.D.B. Paice and F. Wirth. Robustness of nonlinear systems subject to timevarying perturbations, In F. Colonius et al. (eds.), Advances in Mathematical Systems Theory, Birkhäuser, Boston, 2000. To appear.

16. P. Soravia, Optimality principles and representation formulas for viscosity solutions of Hamilton-Jacobi equations, I: Equations of unbounded and degenerate control problems without uniqueness, Adv. Differ. Equ., 4 (1999), 275-296.

17. L.N. Trefethen, A.E. Trefethen, S.C. Reddy and T.A. Driscoll, Hydrodynamic stability without eigenvalues, Science 261 (1993), 578-584.

18. A. Vannelli and M. Vidyasagar. Maximal Lyapunov functions and domains of attraction for autonomous nonlinear systems. Automatica, 21 (1985), 69-80.

19. F.W. Wilson. The structure of the level surfaces of a Lyapunov function. $J$. Differ. Equations 3 (1967), 323-329.

20. V.I. Zubov, Methods of A.M. Lyapunov and their Application, P. Noordhoff, Groningen, 1964. 